\title{
La protección de las mujeres en su vejez
}

\section{Women's economic protection in old age}

\section{\{ Stefano Farné \}** $\{\text { Paola Ríos }\}^{* * *}$}

Presentado: I de agosto de 20I 7. Aceptado: 3I de octubre de 2017.

** MSc. en Economía de la Universidad de Londres, Reino Unido. Director del Observatorio de Mercado de Trabajo y la Seguridad Social de la Universidad Externado de Colombia.

**** Magíster en Economía de la Universidad Externado de Colombia. Estudiante del pre-master en Econometría de la Universidad de Tilburg.

Los autores agradecen a Hugo Cifuentes, integrante de la Comisión Asesora Presidencial sobre el Sistema de Pensiones en Chile, por sus aportes al documento. Dor: https://doi.org/IO.I860I/25390406. $\mathrm{n} 2.03$ 


\section{RESUMEN}

En Colombia, como en otros países del mundo, las mujeres reciben menores salarios y experimentan mayores interrupciones en el mercado formal de trabajo, frente a los hombres. Las anteriores condiciones reducen la probabilidad de tener una protección económica adecuada en la vejez. La distribución inequitativa de las labores no remuneradas del cuidado del hogar desempeña un rol importante en este contexto. Por ello, las políticas encaminadas a corregir las disparidades de género en el mercado laboral son esenciales. El presente artículo reconoce la importancia de estas acciones. Sin embargo, se concentra en políticas directamente relacionadas con mecanismos de protección social que mejoren la probabilidad de que las mujeres reciban una pensión o de que incrementen su mesada pensional. El artículo describe y explica las bajas tasas de cobertura pensional entre las mujeres adultas mayores colombianas. Adicionalmente, explora y analiza las políticas de protección en favor de las mujeres que se han llevado a cabo en otros países con el propósito de discutir posibles iniciativas para Colombia.

\section{Palabras clave:}

Pensiones; seguridad social; género.

\section{Abstract}

In Colombia, as in many other countries, women earn lower wages and experience more intermittency in the formal labor market compared to men. The last conditions reduce the likelihood of having an adequate economic protection in old age. The unequal distribution of unpaid household and child care plays a major role in this context. Hence, policies aimed at redressing gender disparities in labor market are essential. This article acknowledges the relevance of the latter actions. However, this study focuses on policies directly related with social protection mechanisms that improve women's probability of receiving a pension or increase the pension payment. This article provides a description of the low pension coverage among Colombian older women. Furthermore, the paper explores and analyses policies conducted in other countries, in order to discuss possible initiatives for Colombia.

\section{KEYWORDS:}

Pensions; social security; gender. 


\section{INTRODUCCIÓN}

La evidencia empírica internacional ha mostrado cómo, en promedio, la vida laboral de las mujeres se caracteriza por periodos de inactividad más largos, menores tasas de ocupación, menores ingresos y más empleos de tiempo parcial que los de los hombres ${ }^{\mathrm{I}}$.

Es evidente que las anteriores circunstancias comprometen las posibilidades de las mujeres de recibir una pensión económicamente suficiente en la edad de retiro y, a menudo, hasta de calificar para obtener el derecho a una pensión contributiva, aunque sea, mínima.

Algunas de las comentadas condiciones laborales derivan de distorsiones y discriminaciones ajenas a un correcto funcionamiento del mercado de trabajo y, por tal razón, deberían ser contrarrestadas con políticas de corte laboral. Tal es el caso de las políticas dirigidas a reducir los diferenciales salariales de género o a favorecer una más equitativa redistribución entre oferta de trabajo y cargas familiares entre las parejas en edad activa.

Otras son consecuencias de la función reproductiva de las mujeres y del hecho de que ellas dedican tiempos más largos a las tareas de los hogares y a la atención de otras personas. De hecho, el trabajo de cuidado, a pesar de generar grandes beneficios para la sociedad, generalmente, no es remunerado ni reconocido como una actividad económica y, por esta razón, no genera prestaciones pensionales. En este caso, para mejorar la protección de las mujeres en edad avanzada puede mejor recurrirse a medidas que afecten el diseño de los sistemas mismos de pensiones.

El presente documento pretende aportar una contribución a este segundo tipo de soluciones. En la actualidad en el país se está discutiendo la necesidad de una reforma pensional. Hasta ahora esta discusión se ha centrado en el problema de la sostenibilidad financiera del régimen de reparto público y no ha tocado cuestión alguna relacionada con la igualdad de género y la protección de la mujer en la vejez. Así que un objetivo no secundario de este artículo es también insinuar la necesidad de introducir estos temas en la agenda del debate.

$\mathrm{El}$ artículo comprende seis secciones, de las cuales la primera es esta introducción. La segunda recuerda de forma sucinta las condiciones para pensionarse tion of Older Women" (Discussion paper I, UN Women, 20 I 5); Verónica Amarante, Maira Colacce y Pilar Manzi, La brecha de género en jubilaciones y pensiones, los casos de Argentina, Brasil, Chile y Uruguay, Serie: Asuntos de Género (Santiago de Chile: cepal y Cooperación Española, 20r6); Estelle James, "Gender in the (Nonfinancial) Defined Contribution World: Issues and Options," en Nonfinancial Defined Contribution Pension Schemes in a Changing World, volume 2: Gender, Politics and Financial Stability, editado por Robert Holzmann, Edward Palmer y David Robalino (Washington D.C.: The World Bank, 2013), 3-33. 
en Colombia en los dos regímenes de prima media y capitalización individual. La sección tres relaciona la condición de mujer con sus posibilidades de obtener una pensión de jubilación contributiva. Un cuarto capítulo reseña las medidas existentes en el sistema pensional colombiano dirigidas a mejorar la protección de las mujeres en la vejez. Un quinto capítulo describe unas opciones adicionales de protección que se han propuesto e implementado a nivel internacional. Por último, una última sección retoma los argumentos desarrollados a lo largo del documento y trae algunas conclusiones de política.

\section{Sistema contributivo de pensiones en Colombia}

El Sistema General de Pensiones en Colombia se compone de dos regímenes excluyentes entre sí: el Régimen de Ahorro Individual con Solidaridad (RAIS) y el Régimen de Prima Media (RPM).

En el primero, los afiliados son titulares de una cuenta de ahorro individual en la cual se depositan sus aportes mensuales con los respectivos rendimientos y que es manejada por unas entidades financieras privadas denominadas administradoras de fondos de pensiones (AFP).

El RPM es un régimen en el que se constituye un fondo común de carácter público, al cual se dirigen las cotizaciones de los afiliados. Este fondo es administrado por Colpensiones, una empresa industrial y comercial del Estado.

Las contribuciones a cualquiera de los regímenes son de carácter obligatorio para los trabajadores y empleadores, pero son los primeros quienes eligen en qué sistema cotizar.

Los cotizantes del RPM obtienen una renta vitalicia conforme a unos requisitos previamente definidos en la ley, de ahí que también se denomine régimen con prestaciones definidas. En especial, los cotizantes deben cumplir con los requisitos de la edad (62 años para los hombres y 57 años para las mujeres) y las semanas cotizadas ( 1300 ) para obtener una pensión.

Por el contrario, el RaIs es un sistema de contribuciones definidas, donde los aportes de los afiliados se relacionan de forma más directa a los beneficios obtenibles los cuales no son garantizados a priori. De hecho, el otorgamiento de la prestación depende de que la persona logre acumular un ahorro suficiente para financiar una pensión del i i० \% del salario mínimo. En este régimen, la edad de jubilación se vuelve vinculante solo cuando el afiliado decide retirar todos sus ahorros - en el caso en que ellos, con sus respectivos rendimientos, no alcancen un monto suficiente para financiar una pensión mínima- o solicite hacer efectiva la garantía de pensión mínima. Más precisamente, el RaIs permite que al afiliado, que durante su vida activa no acumule un capital suficiente para financiar una pensión de salario mínimo y que al mismo tiempo cuente con la edad de pensión 
y I 50 semanas cotizadas, solicite la devolución total de los saldos ahorrados o, si es carente de ingresos, que se le otorgue una renta vitalicia igual a un salario mínimo con cargo al fondo de garantía de pensión mínima².

En ambos regímenes, en caso de muerte del titular de la pensión, esta se revierte en su totalidad a los sobrevivientes habientes de derecho.

\section{Mujeres colombianas: misma tasa de cotización, Pero menos Pensiones QUE LOS HOMBRES}

Durante su vida activa, las mujeres colombianas mantienen tasas de cotización al sistema pensional similares a las de los hombres de 37, I \% y 37,2 \%, respectivamente a 20r6. Sin embargo, cuando se investiga su condición de pensionadas, resalta el hecho de que ellas presentan una menor cobertura y mesadas de montos inferiores. Así, mientras que el 30,9\% de los hombres en edad de retiro recibe una pensión (de jubilación, invalidez o sobreviviente), solo el 20,9 \% de las mujeres lo hace. Y si se consideraran solo las pensiones de jubilación —es decir, las que se originan en un ahorro contributivo previo-, esta brecha sería aún mayor, en vista de que las mujeres son las principales beneficiarias de la pensión de sobrevivientes ${ }^{3}$. En cuanto a los ingresos, la mesada pensional promedio de las mujeres es el $80 \%$ de la mesada pensional promedio de los hombres 4 .

Si las brechas de cobertura pensional entre hombres y mujeres son más amplias que las brechas en la cotización a pensiones, dos aspectos pueden ser pertinentes para explicar esta discrepancia. En primer lugar, puede que haya diferencias en las densidades de cotización, como también puede ser que sea el mismo diseño de los esquemas de jubilación el que afecte de manera diferente a hombres y a mujeres.

La densidad de cotización es el porcentaje de la vida laboral de las personas en la que se hacen aportes al sistema de pensiones. Este parámetro es particularmente importante en el RPM, pues la consecución de una pensión está sujeta a que las personas coticen por lo menos I 300 semanas al sistema.

En el RAIS un menor tiempo de cotización podría compensarse con mayores ingresos o mayores rendimientos. No obstante, mayores periodos de cotización aumentan la probabilidad de obtener una pensión y la densidad de

2 Colombia, Decreto (832 de I996), sobre la reglamentación parcial de la Ley Ioo de i993, art. 3.

3 Según información de la Superintendencia Financiera, el 35,9\% de las pensiones que reciben las mujeres en Colombia son de sobrevivientes, en el caso de los hombres este porcentaje es mucho menor I $0,7 \%$.

4 Todas las anteriores estadísticas son promedios del 20 r 6 que se elaboraron con base en la Gran Encuesta Integrada de Hogares del Departamento Administrativo Nacional de Estadística (DANE) y se refieren a todo el territorio nacional. 
cotización cobra importancia en el caso de la garantía de pensión mínima, la cual exige un periodo de aporte mínimo de i 50 semanas.

Las mujeres tienen una mayor probabilidad de tener periodos más largos de inactividad o desempleo, lo que genera que sus historias laborales tengan más interrupciones y, por ende, que sus densidades de cotización sean menores. Este no es un fenómeno exclusivo de Colombia. Por ejemplo, la Organización para la Cooperación y el Desarrollo Económicos (OCDE) ${ }^{5}$ estima que en los países europeos una mujer de dieciocho años tiene una expectativa de vida laboral de tres años menos que los hombres.

López y Lasso ${ }^{6}$ evalúan las diferencias por sexo en los flujos de los trabajadores entre los estados de (in)actividad para Colombia. Los autores encuentran que mujeres en diferentes condiciones familiares y de formación ${ }^{7}$ tienen una mayor probabilidad de mantenerse en la inactividad que los hombres jefes de hogar. De igual manera, la probabilidad de transitar del desempleo a la inactividad es superior para las mujeres.

Las responsabilidades familiares parecen ser un importante determinante para explicar periodos más extensos en la inactividad por parte de las mujeres. De las mujeres inactivas en el 2016 que declararon haber trabajado, cerca de I,9 millones (el 4I,6\%) sostuvo haber dejado su empleo por atender responsabilidades familiares. En contraste, en el caso de los hombres el porcentaje que refirió a las responsabilidades familiares como la razón por la cual dejó de trabajar es solo del 2,5\%, es decir unas 45 mil personas. Asimismo, de las mujeres inactivas que declararon haber dejado de buscar trabajo, casi cinco millones afirmaron que lo hicieron por las responsabilidades familiares $(49,4 \%)$. Nuevamente, el contraste con los hombres es bastante desigual, pues apenas 45 mil de ellos $(2,3 \%)$ identifica a las responsabilidades familiares como la razón principal para dejar de buscar trabajo.

En el caso del desempleo, también hay grandes diferencias entre hombres y mujeres. Por ejemplo, en el 2016 , el tiempo promedio que hombres y mujeres demoraron para conseguir su último trabajo fue mayor para las mujeres. El gráfico I además de mostrar que las mujeres tardan unos meses más que los hombres en conseguir un empleo, también indica que esta diferencia se incrementa con la edad.

5 Organisation for Economic Co-operation and Development (oEcD), Pensions at a Glance 20I5: OECD and G2o indicators (París: oecd Publishing, 20 I 5).

6 Hugo López y Francisco Lasso, "Diferencias por sexo en los flujos de trabajadores entre estados laborales y el futuro laboral de las mujeres colombianas", en Desempleo femenino en Colombia, eds. Luis Eduardo Arango, Francesca Castellani y Eduardo Lora (Bogotá: Banco de la República y BID, 20r6), $29-65$.

7 Se tienen en cuenta si las mujeres son cónyuges o jefes de hogar, si tienen solo primaria o si tienen formación técnica o tecnológica, también se desagrega por si tienen hijos menores de once años. 


\section{Gráfico 1. \\ Brechas en el tiempo promedio (meses) de búsqueda de trabajo, por edad. \\ Total nacional. Promedio anual 2016}

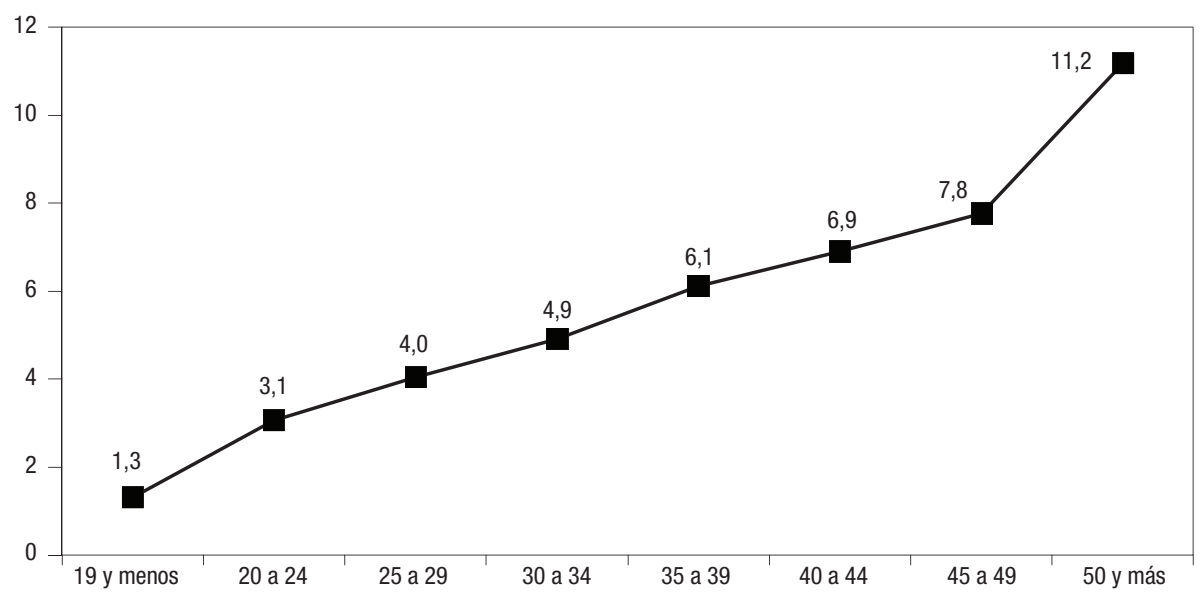

Fuente: cálculos de los autores con base en la GEIH. Se excluyeron a los que reciben una pensión.

La inequitativa asignación de las cargas familiares y las del hogar parece ser un determinante para entender por qué las mujeres presentan una mayor probabilidad de tener periodos más extensos de desempleo. Arango y Ríos ${ }^{8}$ concluyen que la duración del desempleo es superior para las mujeres casadas o en unión libre, que para los hombres con el mismo estado civil. Estos resultados se mantienen para todos los niveles de ingreso de la pareja. Y como había que esperar, la presencia de niños en el hogar tiene un efecto mayor en la duración del desempleo de las mujeres que en la de los hombres.

Monroy y Olarte9 estiman con la Encuesta Nacional del Uso del Tiempo 2OI2-2OI3, que en hogares conformados por parejas que participan en el mercado laboral, los hombres solo dedican una hora diaria a las labores domésticas no remuneradas, mientras que las mujeres dedican en promedio cuatro horas a estas actividades. En contraste, en las actividades remuneradas las mujeres dedican 7, I horas y los hombres 8, I horas al día. Es decir, las mujeres participan menos en las actividades remuneradas y en una proporción más alta que los hombres en las labores del hogar que no se remuneran al interior del hogar o fuera de este.

8 Luis Eduardo Arango y Ana María Ríos, "Duración del desempleo en Colombia: género, intensidad de búsqueda y anuncios de vacantes," en Desempleo femenino en Colombia, eds. Luis Eduardo Arango, Francesca Castellani y Eduardo Lora (Bogotá: Banco de la República y BID, 20I6), I35-г69. en el hogar: particularidades de género para Colombia”, en INVESTIGAS, siete estudios realizados a partir de la encuesta nacional de uso del tiempo, Colombia 20I2-20I3 (Bogotá: DANE y UNFPA, 2OI5). 
En resumen, a raíz de su mayor contribución a las tareas del hogar y de cuidado de otras personas, las mujeres tienen una menor densidad de cotización y esto lleva a una menor probabilidad de calificar para una pensión de jubilación. Adicionalmente, esta probabilidad puede resultar perjudicada por unos efectos indirectos, especialmente, en los regímenes de contribuciones definidas.

Por ejemplo, los periodos fuera del mercado laboral pueden traer pérdidas en el capital humano y social de las mujeres, las cuales son determinantes para aspirar a mejores oportunidades laborales, mejores ingresos ${ }^{10} \mathrm{y}$, en últimas, para obtener una pensión.

En el régimen de contribuciones definidas colombiano, RAIs, la menor densidad de cotización tiene un ulterior efecto negativo sobre el monto y las posibilidades de pensionarse de las mujeres, pues a la cuenta de los afiliados inactivos se cobra una comisión adicional a la que ya se pagó cuando se aportó como ocupado, por concepto de la administración de los recursos. Esta comisión puede ser como máximo del 4,5\% sobre los rendimientos abonados durante el mes en la cuenta individual ${ }^{11}$. En vista de que las mujeres tienen una mayor probabilidad de tener periodos más largos de cesantía e inactividad, esta comisión afecta la acumulación del capital para financiar sus futuras pensiones ${ }^{\mathrm{I2}}$.

$\mathrm{El}$ anterior es un ejemplo de cómo los diseños de los sistemas de pensiones, junto con las diferencias en las densidades de cotización de hombres y mujeres, son relevantes para entender las brechas de protección económica en la vejez.

Otros elementos de diseño importantes en el sistema pensional colombiano son las tablas de mortalidad unisex, o diferenciadas por género, la edad de retiro y las pensiones de sobrevivientes. Sus efectos sobre las pensiones de las mujeres se discutirán en las siguientes secciones.

Julie Hotchkiss y Melinda Pitts, "The Role of Labor Market Intermittency in Explaining Gender Wage Differentials", Am. Econ. Rev. 97, n. ${ }^{2}$ (2007): 4I 7-42 I; Joyce P. Jacobsen y Laurence M. Levin, "Effects of Intermittent Labor Force Attachment on Women's Earnings", Month. Lab. Rev. I I8, (I995): I4-I9.

$\mathrm{Y}$ en todo caso no puede exceder el valor que resulte de aplicar al último ingreso base de cotización del afiliado cesante el $50 \%$ del porcentaje de comisión de administración que se encuentre cobrando la administradora a sus afiliados cotizantes. Esta comisión se cobra a los trabajadores dependientes que culminen su contrato y a los independientes que dejen de cotizar durante tres meses.

Cabe recordar, sin embargo, que a los desempleados, que como asalariados cotizaron un año durante los últimos tres o que como independientes cotizaron dos años durante los últimos tres a una Caja de Compensación Familiar, es posible acudir al mecanismo de protección al cesante el cual se hace cargo del pago de las cotizaciones al sistema pensional por un periodo máximo de seis meses y sobre un ingreso de un salario mínimo si los beneficiarios muestran buscar activamente un empleo o se capacitan. 


\section{Medidas que actualmente Se aplican para mejorar LA PROTECCIÓN DE LAS MUJERES EN LA VEJEZ}

Dadas las mayores dificultades que enfrentan las mujeres para poderse jubilar, los sistemas pensionales han incorporado medidas tendientes a mejorar la protección y mantener los niveles de vida de las mujeres en la vejez.

En el caso de Colombia, se han identificado cuatro medidas principales: la licencia de maternidad, la pensión de sobrevivientes, la adopción de tablas de mortalidad unisex y la posibilidad de pensionarse en edades más tempranas. Las cuatro se analizan a continuación.

\section{LICENCIA DE MATERNIDAD}

La licencia de maternidad es una prestación que permite a las mujeres interrumpir sus actividades laborales para dedicarse al cuidado de los hijos recién nacidos, manteniendo sus remuneraciones y sus contribuciones al sistema de pensiones.

A partir de enero del 2017 , a las mujeres colombianas se les reconoce un periodo de licencia por maternidad de dieciocho semanas ${ }^{13}$. Durante este periodo, las madres tienen derecho a una remuneración mensual igual al salario que devengaban al momento de iniciar su licencia. Si el salario es variable, se toma como referencia el promedio del último año de servicio o de todos los meses trabajados si el tiempo de servicio fuera inferior ${ }^{\mathrm{I}}$.

Además, la legislación colombiana contempla una protección especial a las madres trabajadoras para que no puedan ser despedidas durante el embarazo ni en los tres meses posteriores al parto ${ }^{15}$.

El pago de la licencia de maternidad se financia con el Fondo de Solidaridad y Garantía del Sistema de Salud. Para acceder a esta prestación las mujeres deben haber cotizado al sistema de salud durante el periodo de gestación sin interrupción. En caso de que no se haya completado el tiempo de cotización requerido, la Corte Constitucional ${ }^{16}$ ha estipulado que, si las semanas no cotizadas fueran inferiores a las diez, la madre tiene derecho a la licencia en su totalidad; si

I3 El número de las semanas de licencia de maternidad se ajusta al mínimo de semanas sugeridas en la Recomendación n. ${ }^{\circ}$ I9I del 2000 de la Organización Internacional del Trabajo. En América Latina, Colombia junto con Brasil, Chile y Venezuela son los países que otorgan los periodos más extensos de licencia de maternidad, de dieciocho semanas y en Brasil de casi diecinueve. Para el resto de países latinoamericanos la licencia varía entre seis, cuatro y trece semanas.

I4 Colombia, Código Sustantivo de Trabajo, sobre la regulación de derecho individual del trabajo de carácter particular, y las de derecho colectivo del trabajo, oficiales y particulares, art. 236.

I 5 Código Sustantivo de Trabajo, art. 239. A pesar de ello, los despidos son frecuentes. De acuerdo con la Encuesta de Demografía y Salud (2O I 5) el 4 \% de las mujeres entre I 3 y 49 años afirmaron que fueron despedidas estando embarazadas. 
por el contrario fueran diez o más se le debe reconocer una licencia proporcional al tiempo cotizado ${ }^{17}$.

Por su lado, las mujeres continúan aportando al sistema de pensiones (y salud) por el porcentaje que les corresponde por ley como trabajadoras independientes o empleadas, según el caso, y los empleadores siguen cotizando la parte a su cargo sobre el valor de la licencia ${ }^{18}$.

Para las mujeres fuera del sistema contributivo de salud, no existe un esquema alternativo que brinde protección económica durante la maternidad. Sin embargo, a estas mujeres se les brinda un acceso gratuito a los servicios de salud.

En resumen, la licencia de maternidad en Colombia permite a las mujeres mantener sus ingresos y seguir ahorrando durante dieciocho semanas para su vejez. Sin embargo, la licencia de maternidad puede convertirse en una causa de discriminación en el mercado laboral y esto, a su vez, repercute de modo negativo en la capacidad de las mujeres de ahorrar y lograr una pensión en la vejez.

De acuerdo con Rossin-Slater ${ }^{19}$, a nivel teórico, los impactos de la licencia maternidad sobre el empleo y los salarios de las mujeres son ambiguos. Por un lado, la licencia puede garantizar a las mujeres una continuidad en el mercado de trabajo, lo cual mejora sus perspectivas laborales en el futuro. Por el otro, la existencia de la licencia puede cambiar las preferencias de las mujeres, haciendo que algunas tomen un tiempo mayor al mínimo garantizado y ello puede generar efectos negativos sobre los salarios y las perspectivas laborales de las mujeres. Además, los empleadores pueden considerar la licencia costosa y discriminar a las mujeres reconociéndoles menores salarios o contratando preferiblemente a los hombres.

En la práctica, los impactos de las licencias de maternidad en el mercado laboral dependen de varios factores incluyendo su duración, si es remunerada o no y si se contempla una protección especial contra el despido. En términos generales y para los países desarrollados, Rossin-Slater ${ }^{2 \circ}$ sostiene que cuando la licencia es menor a un año puede haber una mejora en la continuidad laboral de las mujeres; las licencias que tengan extensiones superiores al año pueden tener efectos negativos sobre los ingresos, el empleo y en el avance en las carreras laborales de las mujeres.

I 7 En muchos casos es necesario recurrir a unatela para que se otorgue una licencia sin cumplir con el requisito de tiempo de cotización, pues la norma indica que es el empleador quien debería asumir el costo de la licencia de maternidad.

Colombia, Decreto (I406 de I999), sobre la adopción de disposiciones reglamentarias de la Ley roo de I993, art. 40.

I9 Maya Rossin-Slater, "Maternity and Family Leave Policy” (Iza Discussion Paper ı 50o, Bonn, January 20I7). 
Con referencia al caso colombiano Ramírez, Tribín y Vargas ${ }^{21}$ reseñan numerosos estudios que sugieren consecuencias negativas de los costos asociados a una eventual maternidad —incluida la licencia de maternidad - sobre la empleabilidad de las mujeres.

En Colombia, los hombres también tienen derecho a una licencia de paternidad en ocasión del nacimiento de un hijo. Esta es voluntaria y tiene una duración de ocho días hábiles. Para valerse de esta licencia, el padre debe haber cotizado durante los meses correspondientes al periodo de gestación de la madre.

\section{TASA DE REEMPLAZO (EN EL RPM)}

En el RPM colombiano, el valor de la mesada pensional depende del ingreso base de liquidación - es decir, el promedio de los ingresos sobre los cuales se ha cotizado durante los diez años anteriores al reconocimiento de la pensión actualizados por el índice de precios al consumidor - y de la tasa de reemplazo —es decir, el porcentaje del ingreso base de liquidación que se reconoce como pensión- según la fórmula que establece el artículo io de la Ley 797 del 2003 y que es idéntica para ambos géneros.

Lo anterior lleva a que personas con el mismo ingreso base de liquidación y el mismo número de años cotizados al sistema tengan derecho a una misma pensión, independiente de su edad y su género. Por tanto, la temprana edad de jubilación y la más alta expectativa de vida no constituyen un factor perjudicial en la determinación de las mesadas pensionales de las mujeres. El valor de la mesada es igual para hombres y mujeres, dado que se fija exclusivamente con base en la fórmula de la tasa de reemplazo que varía entre el $55 \%$ y el 70,5\%, dependiendo del ingreso básico de liquidación y de las semanas cotizadas a partir de las I300. De igual manera, se garantiza una pensión de salario mínimo, cuando al aplicar la tasa de remplazo la mesada resultante sea inferior a un salario mínimo.

En la práctica, la fórmula de la tasa de reemplazo de los sistemas de reparto conlleva implícita la aplicación de tablas de mortalidad idénticas para hombres y mujeres. Como veremos, este no es el caso de los regímenes de capitalización, incluido el raIs colombiano.

\section{EdAd DE RETIRO (EN EL RPM)}

En Colombia, las mujeres pueden jubilarse cinco años más temprano que los hombres, al cumplir los 57 años de edad. 
Una diferencia en la edad de jubilación en favor de las mujeres era muy común en los regímenes pensionales de beneficios definidos. Se consideraba un reconocimiento a las labores del hogar y del cuidado de terceras personas a las cuales las mujeres se dedicaban en su mayoría. También porque así una pareja de trabajadores terminaba pensionándose al mismo tiempo, siendo, en general, la mujer más joven que el hombre.

El RPM colombiano favorece a las mujeres al combinar la utilización (implícita) de tablas de mortalidad unisex con la posibilidad de pensionarse cinco años antes que los hombres, con tal de haber cotizado por lo menos 25 años al sistema.

Por lo dicho en la sección anterior, es evidente que si un hombre y una mujer cumplen los requisitos etarios para lograr una pensión, y si ambos tienen periodos de cotización y salarios similares, en el momento en que lleguen a solicitar su pensión ambos tendrán la misma mesada pensional y las mujeres disfrutarán en promedio cinco años más de su jubilación.

En años recientes, la mayor participación de la mujer en el mercado del trabajo, la menor fecundidad y el mayor - aunque lento- involucramiento de los hombres a la economía del cuidado del hogar han traído una tendencia mundial hacia la equiparación entre géneros de la edad de retiro y la temprana edad de jubilación ha sido reemplazada por créditos pensionales por maternidad.

Vale la pena resaltar que una temprana edad de jubilación tiene efectos muy diferentes en el rais. Ceteris paribus, al ampliarse el periodo de disfrute de la pensión, la consecuencia será que las mujeres tendrán menores mesadas pensionales.

El efecto conjunto de una temprana edad de jubilación y de la aplicación de tasas de mortalidad específicas puede tener consecuencias devastadoras sobre las pensiones de las mujeres en el RAIs. El gráfico 2 compara la tasa de reemplazo que garantiza el RPM y que implícitamente reconoce el RAIs a una mujer de 57 años que decide pensionarse después de haber cotizado por I 300 semanas a una tasa de I I, 5 \% sobre unos ingresos expresados en múltiplos de un salario mínimo vigente.

Se plantean dos opciones con respecto a la tasa de rendimiento real de los ahorros que reconoce la AfP a la cual está afiliada la mujer: 4 \% (sección A) y $6 \%$ (sección B) constantes durante los 25 años. En el primer caso, si la mujer ha cotizado toda su vida activa sobre un ingreso, por ejemplo, de cuatro salarios mínimos, el RPM le reconocería una tasa de reemplazo del 63,7\%, mientras que en el RaIs sería apenas del $27,5 \%$. Si la tasa de rendimiento sube al $6 \%$, la diferencia se reduce, pero sigue muy significativa. En el RPM la tasa de remplazo no cambia al modificarse la tasa de rendimiento y siempre será del $63,7 \%$ y en el RAIs alcanzaría el 43,7\%. En cualquier caso, la mesada pensional resultaría 
sustancialmente inferior en el RAIS que en el RPM a menos que las AFP no reconozcan a sus afiliados rendimientos muy altos, muy por encima de los que han reconocido en los últimos años.

\section{Gráfico 2.}

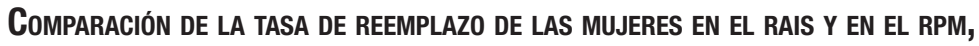
SEGÚN DIFERENTES NIVELES DE IBC

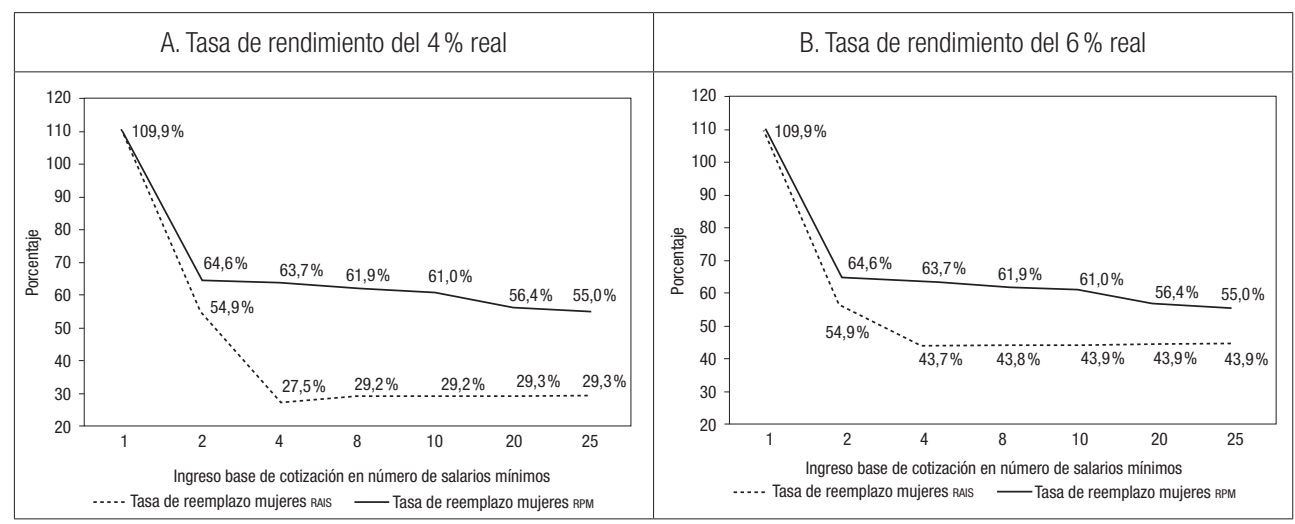

Estos cálculos se hicieron bajo el supuesto de que la mujer no tiene beneficiarios y empieza a cotizar a la edad de 25 años.

Fuente: modelo de simulación pensional elaborado por el Observatorio del Mercado de Trabajo y la Seguridad Social de la Universidad Externado de Colombia

\section{Pensión de SOBReVIVIentes}

Las pensiones de sobrevivientes "constituyen uno de los más importantes instrumentos de política para la protección de las mujeres que carecen de una pensión de jubilación propia”22.

En Colombia, del total de las pensiones de sobrevivencia entregadas por ambos regímenes, el $80 \%$ es disfrutado por las mujeres ${ }^{23}$. Esto se debe a que las expectativas de vida y la composición de los hogares propician que sean las mujeres las que tengan una mayor titularidad sobre este tipo de pensiones. Pues las mujeres tienen una mayor expectativa de vida al nacer (79 años), que la de los hombres (73 años) ${ }^{24}$ y son estos últimos quienes en una mayor proporción reciben pensiones de jubilación. Además, en los hogares biparentales, en los que

22 Camila Arza, "The gender dimensions", 29.

23 Cálculo elaborado con la información de la Superintendencia Financiera con fecha de corte a diciembre de 2016 .

24 Colombia, DANE, Conciliación censal i985-2020. 
el hombre es el cabeza de hogar ${ }^{25}$, la compañera, en promedio, es menor 4,3 años que su pareja y, por tanto, tiene mayor probabilidad de ser la sobreviviente.

El sistema de sobrevivientes colombiano es muy generoso, pues otorga al cónyuge que queda viudo el $100 \%$ de la pensión del fallecido ${ }^{26}$. A nivel mundial, este porcentaje es mucho menor y varía entre un $40 \%$ y un $80 \%$, siendo el promedio de $64 \%^{27}$, de acuerdo con el número de dependientes, los ingresos y la edad del sobreviviente.

Según la ley colombiana, si el beneficiario es mayor de treinta años al momento de la titulación recibe la pensión de manera vitalicia; en el caso en el que su edad sea menor, recibe una prestación mensual de igual monto por veinte años durante los cuales debe contribuir al sistema para constituir su propia pensión.

Vale la pena resaltar que la pensión de sobrevivientes no genera ningún tipo de incompatibilidad si ya se tiene o se va a obtener una pensión de vejez o de invalidez.

\section{LA EXPERIENCIA INTERNACIONAL Y LAS ALTERNATIVAS PARA MEJORAR LA PROTECCIÓN EN LA VEJEZ DE LAS MUJERES ${ }^{28}$}

Esta sección reseña algunos mecanismos introducidos en los sistemas pensionales a nivel internacional que se han diseñado con el propósito de mejorar la protección económica en la vejez de las mujeres y que todavía no han tenido aplicación en Colombia.

Se analizan los créditos pensionales que compensan a las mujeres por dedicar una parte importante de su tiempo a las tareas del hogar y a la atención de otras personas, las tablas de mortalidad unisex, cuya introducción en los sistemas de contribuciones definidas permitiría mejorar las mesadas pensionales de las mujeres afectadas por su mayor expectativa de vida, y los fondos previsionales compartidos, así como las pensiones compartidas en caso de divorcio que se

Esta estructura de hogar es el 50\% del total de los hogares. Cálculos propios con base en la GEIH 20 I 6. Cuando hay hijos dentro del hogar, el compañero o compañera reciben el $50 \%$ de la pensión y los hijos el otro $50 \%$ restante. Los hijos pueden recibir la pensión de sobrevivientes hasta los i 8 años o hasta los 25 años cuando tengan dependencia económica y estén estudiando y la pueden recibir de manera indefinida si tienen una condición de invalidez.

Mariano Bosch, Solange Berstein, Francesca Castellani et al., "Diagnóstico del sistema previsional colombiano y opciones de reforma" (Nota Técnica 825, Unidad de Mercados Laborales y Seguridad Social, BID, junio de 2015). Children: The Role of Credits Within Pension Systems in oEcD and Eu Countries", en Nonfinancial, editado por Holzmann, Palmer y Robalino, 75-I Io; oecd, Pensions at a Glance, y Comisión Asesora Presidencial Sobre el Sistema de Pensiones-Comisión Bravo. Comisión Presidencial Pensiones: informe final (septiembre de 20 I 5); fuentes de las cuales constituye un resumen y de las cuales recoge sus principales hallazgos. 
basan en el principio de que la pensión deja de ser un derecho individual para volverse un derecho conjunto de la pareja.

\section{Créditos pensionales por maternidad y CUIDAdo}

Son beneficios que se conceden para compensar el tiempo dedicado al cuidado de terceros y que pueden consistir en el reconocimiento de periodos de cotización, de montos de dinero que se acreditan al ahorro pensional, en el no computo del tiempo de inactividad o de bajos ingresos a la hora de calcular el ingreso de liquidación de la pensión o en la concesión de mayores pensiones dependiendo del tipo de esquema pensional considerado.

Los más utilizados son los créditos pensionales por maternidad, de los cuales se hacen acreedoras las mujeres que tienen hijos.

En Italia, por ejemplo, el valor de la pensión que se otorga depende de un coeficiente "de conversión" ${ }^{29}$ que disminuye (aumenta la pensión) con los años de la persona, premiando así a quienes aplacen su edad de retiro. Bajo el anterior esquema a las madres que tengan uno o dos hijos se les acredita un año de edad y a las que tengan tres o más hijos se les acreditan dos. De esta forma, las mujeres se benefician con una pensión más generosa, dado que el cálculo de la pensión se realizará como si se hubiesen retirado uno o dos años más tarde.

En Alemania, la definición de una pensión se basa en un sistema de puntos. En este esquema se reconoce un punto cuando los ingresos anuales del afiliado corresponden a los ingresos promedios nacionales y, de forma proporcional, se otorga más o menos de un punto según si los ingresos superan o son inferiores al ingreso promedio. Los créditos de maternidad acreditan a uno de los padres tres puntos por hijos nacidos antes de 1992 y dos puntos si nacieron después de 1992.

En América Latina, Uruguay, Chile y Bolivia tienen esquemas de créditos pensionales por maternidad. En el caso de Uruguay, cuando las mujeres alcanzan la edad de jubilación (sesenta años) tienen derecho a computar al sistema pensional un año por cada hijo nacido vivo o adoptado, con un máximo de cinco $^{30}$. En la práctica, lo anterior permite a las progenitoras pensionarse con un menor número de años de cotizaciones — respecto al mínimo legal de treinta años-o aumentar la tasa de reemplazo de su pensión.

En Chile, se reconoce a las mujeres un bono pensional por cada hijo nacido vivo o adoptado igual al ro \% de dieciocho ingresos mínimos mensuales ${ }^{31}$. El valor del bono se establece al momento del nacimiento del hijo, pero se liquida

29 Que convierte el capital acumulado en una pensión de jubilación.

$30 \quad$ Uruguay, Ley ( 8395 de 2008), sobre beneficios jubilatorios.

3 I Chile, Ley (20 255 de 2008), sobre la reforma previsional. En 20 i 6 el bono corresponde a aproximadamente 450000 pesos chilenos, unos dos millones de pesos colombianos. 
con los respectivos rendimientos, cuando la mujer cumple los 65 años. El bono es depositado en la cuenta previsional de la beneficiaria, permitiéndole así mejorar el monto de su pensión.

En Bolivia, a las mujeres ${ }^{2}$ que califican por la Prestación Solidaria de Vejez se les reconoce un beneficio por hijo nacido vivo. A esta prestación se puede acceder cuando se cumplan los $5^{8}$ años de edad, se tengan por lo menos diez años de cotización y cuando lo que se otorgaría por pensión de vejez no supere un máximo establecido.

El beneficio entregado a las mujeres puede tomar dos vías. La primera es que las madres pueden acceder antes a la Pensión Solidaria de Vejez, pues por cada hijo nacido vivo pueden solicitar que se disminuya un año en la edad de acceso (con un máximo de tres años). La segunda vía es que cuando ya hayan cumplido los 58 años, les computen por cada hijo un año de aportes, hasta un máximo de tres. Con esta opción, las mujeres pueden incrementar sus periodos de cotización (y alcanzar los diez años requeridos) o conseguir una mesada pensional más alta (sin superar el máximo establecido).

En muchos países se reconocen también créditos pensionales por el cuidado de menores de edad que incluyen o se suman a los beneficios por maternidad. En estos casos se establece un periodo de cuidado máximo y los beneficios pensionales dependen también de la edad de los niños y del hecho de que las madres-padres trabajen o no. En ocasiones, los reconocimientos pueden estar ligados al salario mínimo (Francia, Polonia), o a un porcentaje de los salarios anteriores (Japón, Eslovaquia, Bélgica), o para acceder a ellos se fija un tope máximo de ingresos, para favorecer a los hogares con menores recursos (Francia).

En Luxemburgo, cuando se finaliza la licencia de maternidad y uno de los padres opta por una licencia no remunerada o una licencia para trabajar de tiempo parcial para acudir al recién nacido, se les acreditan hasta dos años de cotizaciones que pueden extenderse a cuatro, si los hijos son dos o más, o cuando el hijo tiene una condición de discapacidad. El beneficio lo puede tomar uno de los padres o puede ser dividido entre los dos. El ingreso base de contribución corresponde a los ingresos anteriores al momento de la solicitud de la acreditación de estos años.

Los créditos pensionales también se han extendido a reconocimientos por el cuidado de otros miembros del hogar. De forma similar al cuidado de los hijos, son las mujeres quienes en mayor proporción que los hombres se ocupan del cuidado de adultos mayores y otras personas necesitadas y, por tanto, las que más se benefician de este tipo de créditos. prestaciones y beneficios, art. 77 . 
Por ejemplo, en el Reino Unido, se entregan créditos a las personas que dedican veinte horas al cuidado de alguna de las personas que recibe el apoyo del Gobierno, por ejemplo, por tener alguna condición de discapacidad o si se certifica por un profesional autorizado que la persona a la que se está cuidando, efectivamente, requiere de estos servicios. Los créditos que se otorguen bajo este sistema se pueden contabilizar en los esquemas de pensión de jubilación y en la State Second Pension.

En Italia, el trabajador que convive con un familiar anciano con una grave discapacidad certificada tiene derecho a dos años, continuos o discontinuos, de licencia remunerada. El monto de la licencia es igual al salario devengado al momento de la solicitud e incluye el reconocimiento de las correspondientes cotizaciones al sistema pensional. El beneficiario de la licencia puede también solicitar el "anticipo financiero como garantía pensional (APE)", el cual permite, bajo ciertas condiciones de edad, ingreso y años cotizados, pensionarse tres años antes de la edad establecida y recibir durante este periodo una pensión inferior a la que hubiera tenido derecho al cumplir la edad legal de retiro de 66 años para los hombres y 65 para las mujeres (en 2017).

Recientemente, en Chile, la Comisión Bravo ${ }^{33}$ ha propuesto introducir un esquema de bono para los familiares — hombres o mujeres— que se hagan cargo de personas adultas mayores o dependientes. El valor del bono que se reconocería estaría asociado a la remuneración mínima mensual por cada mes certificado de cuidado ${ }^{34}$.

\section{TABLAS DE MORTALIDAD UNISEX}

Una de las consecuencias de la imparcialidad actuarial, es decir, de la estricta relación entre cotizaciones pagadas y beneficios pensionales recibidos, que caracteriza los sistemas de contribuciones definidas, es la adopción de tablas de mortalidad diferenciadas por sexo para el cálculo de las pensiones.

Es evidente que la diferenciación por sexo, dada la mayor longevidad de las mujeres, lleva a que ellas reciban pensiones más bajas, a paridad de capital ahorrado. Esto es lo que pasa, por ejemplo, en Colombia y en los demás sistemas de capitalización existentes en América Latina.

Una forma para obviar el problema y garantizar a las mujeres mejores mesadas pensionales consiste en la utilización de tablas de mortalidad unisex que

La Comisión Asesora Presidencial sobre el Sistema de Pensiones o Comisión Bravo fue conformada en abril del 20I4 con el objetivo de estudiar el Sistema de Pensiones, realizar un diagnóstico sobre el funcionamiento del sistema y elaborar propuestas destinadas a resolver las deficiencias que se identifiquen. El informe final fue presentado en septiembre de 2015. 
suponen una común (promedio) probabilidad de esperanza de vida para hombres y mujeres. Las tablas de esperanza de vida conjunta permiten agrupar el riesgo de longevidad entre sexos y provocan una redistribución del hombre promedio, cuya pensión disminuye, a la mujer promedio, cuya pensión aumenta, respecto a la situación en la cual se aplican tablas especificas por sexo.

En Estados Unidos y la Unión Europea la contratación de rentas vitalicias debe basarse en el uso obligatorio de tablas de mortalidad unisex $^{35}$.

En Chile, el informe de la Comisión Bravo ${ }^{36}$ propuso la introducción de tablas de mortalidad conjunta en el sistema de capitalización. Al respecto, la FIAP ${ }^{37}$ y Fajnzylber ${ }^{3}$ advierten unas dificultades que puede tener la aplicación de este sistema. Anotan que solo sería posible bajo el esquema de renta vitalicia, dado que las modalidades de retiro programado y otras formas de jubilación similares tienen un carácter de propiedad individual y la posibilidad de herencia de estos activos financieros no permitiría la transferencia de recursos de unas cuentas a otras. En consecuencia, y teniendo en cuenta que son los potenciales pensionados quienes eligen la modalidad de jubilación, la medida podría tener un efecto marginal, ya que los hombres serían inducidos a escoger el retiro programado y las mujeres la renta vitalicia. Resaltan también que las tablas diferenciadas implícitamente incorporan el efecto de otras variables que influyen sobre la longevidad — por ejemplo, el estatus socioeconómico-y, por tanto, esta información se perdería.

Por otro lado, si la modalidad de renta vitalicia fuera obligatoria se desincentivaría el ahorro voluntario a pensiones por parte de los hombres. En este caso, además, la eliminación del retiro programado implicaría cambios cuyas consecuencias prácticas son desconocidas, dado que no hay experiencia de países con esquemas pensionales de capitalización individual que usen tablas de mortalidad unisex $^{39}$.

Según los cálculos de la Asociación Chilena de Aseguradores, aplicar las tablas de mortalidad unisex incrementaría en un 9\% la mesada pensional de las mujeres y reduciría en un $7,4 \%$ la de los hombres. Alternativamente, la Aso-

Amarante, Colacce y Manzi, La brecha de género, i6.

Comisión Bravo, Informe final.

Federación Internacional de Administradoras de Fondos de Pensiones (FIAP), "Analysis of the Proposal for Unisex Mortality Tables for Calculating Old-Age Pensions in the Individually Funded System" (Pension Notes I 5 de abril de 2017).

Eduardo Fajnzylber, "Gender Policy and Pensions in Chile", en Nonfinancial, editado por Holzmann, Palmer and Robalino, I I3-I39. El autor retoma los argumentos avanzados por una anterior Comisión Asesora para la Reforma Previsional de 2006 que ya había analizado la propuesta de introducir tablas de mortalidad unisex en Chile.

Fajnzylber, "Gender”, I I 3-1 39. 
ciación propone ${ }^{40}$ que, por medio de recursos del Pilar Solidario, se financie el diferencial entre la mesada pensional que recibiría una mujer con las tablas de mortalidad propias y lo que se le otorgaría aplicando la expectativa de vida de un hombre. El monto resultante se abonaría mensualmente a las pensiones de las mujeres. Bajo este escenario los hombres no tendrían un detrimento en su pensión de manera directa y las mujeres incrementarían su mesada en un ıо\%. Esta iniciativa ha tenido mayor eco y ha sido incluida en un proyecto de ley que "Crea el nuevo ahorro colectivo, aumenta la cobertura del sistema de pensiones y fortalece el pilar solidario", presentado al Congreso el ro de agosto del 20 I 7.

\section{FONDOS PREVISIONALES COMPARTIDOS Y PENSIONES COMPARTIDAS EN CASO DE DIVORCIO}

La lógica de las pensiones de sobrevivencia se basa en el modelo de familia tradicional en el que el solo trabajo del jefe (generalmente el hombre) permitía mantener económicamente el hogar. En los sistemas de beneficios definidos estas pensiones llevan a una implícita transferencia de los contribuyentes núbiles y célibes, a los que están casados y, principalmente, a las mujeres casadas. Las razones de esta transferencia no son obvias, en especial, en los sistemas de contribuciones definidas que se basan en el principio de la imparcialidad actuarial.

Una alternativa a las pensiones de sobrevivencia son las pensiones conjuntas que en el RaIs colombiano corresponden a las rentas vitalicias que los afiliados compran a una compañía de seguro y cuyo valor depende de las características demográficas del titular y de las personas a su cargo.

Otra opción sustitutiva de la pensión de sobrevivientes son los fondos previsionales compartidos antes de la jubilación en los cuales se reparten las cotizaciones de cada uno de los trabajadores entre una cuenta individual propia y otra cuenta individual de la cual es titular el cónyuge. Es evidente que en una perspectiva pensional, los fondos compartidos favorecen el compañero que trabaja menos en el sector formal, generalmente la mujer.

En ambas alternativas, la transferencia en favor de las mujeres que se dedican al cuidado de la familia se mantiene, pero se internaliza al hogar, del marido a la esposa.

La introducción de los fondos pensionales compartidos fue propuesta y estudiada especialmente en Estados Unidos en la década de los ochenta. En general, esta medida no ha logrado tener mucha aceptación por las dificultades de implementación y el elevado costo de transición que puede acarrear ${ }^{41}$. 
Compartir fondos es posible de forma voluntaria en el primer pilar del sistema pensional público sueco - pero no en la porción de capitalización nocional- y según Stahlberg ${ }^{42}$ tampoco ha resultado muy popular entre los afiliados.

En Chile, la Comisión Bravo ${ }^{43}$ propuso que de las cotizaciones para pensiones de cada trabajador se repartieran un $50 \%$ a su cuenta individual y un $50 \%$ a la cuenta individual de su cónyuge o pareja con la que convive. Esta repartición cesaría con la terminación de la relación conyugal. La anterior propuesta, sin embargo, no fue recogida en el proyecto de ley que actualmente busca modificar el sistema de pensiones en este país, ratificándose así las dificultades prácticas que encuentra la implementación de los fondos pensionales compartidos.

Existe otro mecanismo de más simple implementación que sigue la misma filosofía de considerar la pensión como un derecho conjunto y no individual. Se trata de la pensión compartida en caso de divorcio. En Alemania, Canadá, Suiza, Reino Unido, Irlanda y Japón, por ejemplo, una parte de la pensión es transferida a la expareja en caso de separación. El principal inconveniente de esta medida es que si solo hay una persona de la que se derivan los recursos o si el otorgamiento de la pensión depende completamente del ahorro realizado en la edad activa y no hay un esquema en el que se garantice un ingreso básico, dividir los recursos podría generar dos mesadas pensionales inadecuadas ${ }^{44}$.

La Comisión Bravo ${ }^{45}$ en Chile propuso que en los casos de divorcio los jueces realizaran una partición equitativa de los fondos de pensiones. No obstante, esta propuesta tampoco se tuvo en cuenta en el proyecto de ley presentado hace poco al Congreso y por ahora se mantienen las reglas vigentes en las que la división se puede solicitar por vía judicial a manera de compensación, cuando se haya terminado la relación conyugal y uno de los esposos abogue estar en una posición económica menos favorable. La única restricción es que el traspaso que se haga de fondos no exceda el $50 \%$ de los fondos acumulados en el periodo del matrimonio ${ }^{46}$.

MA, 2007); Howard M. Lams, Gayle L. Reznik y Christopher R. Tamborini, "Earnings Sharing in Social Security: Projected Impacts of Alternative Proposals Using the MINT Model”, Soc. Sec. Bul. 69, n. ${ }^{\circ}$ I (2009): I-I 7; Anna Klerby, Bo Larsson y Edward Palmer, "To Share or not to Share: That is the Question", en Nonfinancial, editado por Holzmann, Palmer y Robalino, 39-65.

Ann-Charlotte Stählberg, “Chapter I I. Comment," en Nonfinancial, editado por Holzmann, Palmer and Robalino, 70-73.

43 Comisión Bravo, Informe final.

44 Jongkyun Choi, “The Role of Derived Rights for Old-age Income Security of Women” (París: Employment and Migration Working Papers 43, oECD Social, oecd Publishing, 2006).

45 Comisión Bravo, Informe final.

46 Chile, Ley (20 255 de 2008), arts. 80 y 8 I. 


\section{Conclusiones}

Pese a que las labores de cuidado del hogar y de sus miembros se prolongan por toda la vida, en el sistema pensional colombiano no existen disposiciones que hagan un reconocimiento explícito a este trabajo indispensable, pero no de mercado, con excepción de la licencia de maternidad.

En general, la protección en la vejez de las mujeres, que son las que se hacen cargo de las tareas del hogar, ha quedado relegada a instrumentos que reflejan todavía una distribución de roles en el hogar y fuera de este, que ya no se ajusta a los cambios sociales experimentados por el país.

Es más, la introducción en I 994 del RAIs ha minado la efectividad de algunos de los mecanismos redistributivos que en el RPM favorecían a las mujeres y a la consecución de ingresos estables en su vejez.

En términos generales, los regímenes de contribuciones definidas como el RAIS se caracterizan por una más estrecha relación entre beneficios, por un lado, y contribuciones capitalizadas y edad de retiro (riesgo de longevidad), por el otro. "Esta estructura, a su turno, ha tenido como consecuencia un mayor énfasis en el rol asegurador del sistema pensional y en los individuos más que en la familia como unidad de referencia. [...] (y) [...] la transición de una forma de seguro que descansaba sobre la unidad familiar a uno centrado en la individualidad ha expuesto a las mujeres a mayores riesgos" ${ }^{47}$.

En ambos regímenes pensionales, además, las (interrumpidas) historias contributivas de las mujeres y su (mayor) expectativa de vida tienen el efecto de mermar su probabilidad de calificar por una pensión contributiva en su vejez o de reducir la mesada pensional devengada.

Por otro lado, el aumento de la expectativa de vida de las mujeres - que, por ejemplo, para una mujer de sesenta años habría aumentado en más de seis años, de 20,8 a 27 según las últimas tablas de mortalidad aprobadas por la Superintendencia Financiera en 2008- hace impostergable en Colombia el aumento de la edad de jubilación de la mujer y su equiparación a la de los hombres.

Según la oit, "una edad de jubilación inferior para las mujeres constituye una discriminación formal contra los hombres. Esta diferencia, allí donde todavía existe, está siendo muy cuestionada" ${ }^{4}$.

Este ha sido el caso de numerosos países latinoamericanos. En efecto, del examen de una muestra de veinte de ellos, once ya contemplan una edad de jubi-

Elsa Fornero, "Chapter i r. Comment", en Nonfinancial, editado por Holzmann, Palmer y Robalino, 75-IIO.

48 Organización Internacional del Trabajo-OIT. Seguridad social: un nuevo consenso (Ginebra: ort, 2002), 87 . 
lación idéntica para hombres y mujeres y en dieciséis la edad de jubilación de las mujeres es mayor de lo que establece en nuestro país la legislación colombiana ${ }^{49}$.

Para que sea equitativo, el aumento de la edad de jubilación de las mujeres deberá ser gradual en el tiempo y acompañado por la introducción de créditos pensionales. Inicialmente podría pensarse en créditos por maternidad o bonos por hijo, pero con el tiempo estos créditos deberán extenderse al cuidado de los hijos y de las personas mayores o discapacitadas.

D'Addio $^{50}$ ha estimado que en los países de la unión europea y de la ocDE que tienen créditos pensionales para el cuidado de menores, de no haberse establecidos, las tasas de remplazo de las pensiones de las mujeres serían en promedio tres o siete puntos porcentuales más bajas. Así que el incremento en las tasas de reemplazo como consecuencia de estos créditos "es bastante modesto a todos los niveles de ingreso (siendo Francia, Luxemburgo y Suiza las excepciones)" ${ }^{1}$.

Fajnzylber ${ }^{52}$ estima que el bono por hijo entregado a las madres chilenas aumentaría en un $20 \%$ la pensión promedio de las mujeres. Aun con este incremento la pensión promedio de las mujeres chilenas alcanzaría a constituir apenas un $45 \%$ de aquella promedio de los hombres. En el caso del Uruguay, en 2015 según la Sociedad Silva Santos ${ }^{53}$ un 36,2 \% de las mujeres que accedieron al crédito de maternidad logró obtener una pensión jubilatoria, lo cual no hubiese sido posible sin el cómputo ficto de las semanas que se les otorgaron.

Los sistemas de contribuciones compartidas pueden encontrar dificultades de implementación en Colombia. Si, por ejemplo, estas medidas se aplicaran es muy probable que cuando se dividan los fondos (pensiones) de los cónyuges (excónyuges), en un gran porcentaje de los casos, ninguno logre cumplir los requisitos para una pensión y ante la inexistencia de un pilar básico universal, el resultado sería la desprotección para las parejas (exparejas) en la etapa de vejez.

Naturalmente, una mayor equidad entre hombres y mujeres es también promovida por medidas que alientan a los hombres a participar más en las actividades de cuidado. Por ejemplo, licencias de paternidad más largas que los ocho días permitidos en Colombia o la posibilidad de repartir entre los padres las licencias de maternidad y de cuidado a terceros mejoran las oportunidades de las mujeres en el mercado laboral. Ellas pueden trabajar más horas, acceder a ocupaciones mejor remuneradas que demandan más tiempo y mantener empleos

Stefano Farné, "Pensiones y mujeres", El Espectador, 28 de Febrero de 20 I 5. https://www.elespectador. com/noticias/economia/pensiones-y-mujeres-articulo-54662 I

50 D’Addio, "Pensions," en Nonfinancial, editado por Holzmann, Palmer y Robalino.

5 I D’Addio, "Pensions," en Nonfinancial, editado por Holzmann, Palmer y Robalino, roo.

$5^{2} \quad$ Fajnzylber, "Gender," en Nonfinancial, editado por Holzmann, Palmer y Robalino.

53 Sociedad Silva Santos, "Acciones con perspectiva de género en la seguridad social. Actualización de informe" (Asesoría General en Seguridad Social, Comentarios de Seguridad Social n. ${ }^{\circ}$ I, 20r6). 
de tiempo completo. Así que, extender la licencia de maternidad posparto a los padres puede contribuir a incrementar la empleabilidad de las mujeres, reducir la brecha salarial entre géneros y la discriminación de los empleadores y, de esta forma, pueden mejorar también las perspectivas pensionales de las mujeres ${ }^{54}$.

Además, en Colombia las prácticas discriminatorias en contra de la mujer trabajadora podrían ser contrastadas con la socialización del pago de la seguridad social así de evitar la doble contribución de la cual deben hacerse actualmente cargo los empleadores que deciden reemplazar a sus trabajadoras en licencia de maternidad con personal temporal.

El aumento de la edad de jubilación reduciría las consecuencias negativas derivadas de la aplicación de las tablas de mortalidad diferenciadas por sexo sobre las pensiones de las mujeres afiliadas al RAIs. Alguna medida compensatoria adicional deberá en todo caso ser introducida para subir la tasa de reemplazo de las pensiones de las mujeres en este régimen.

Por último, la figura de pensión de sobreviviente debería ser revisada. Una reducción del porcentaje a recibir de la pensión del difunto y un mecanismo en el que el porcentaje este sujeto a los ingresos que percibe el sobreviviente, harían más equitativo el RPM y en el RAIs se traduciría en pensiones de jubilación más altas para hombres y mujeres.

\section{Bibliografía}

Amarante, Verónica, Maira Colaccea y Pilar Manzi. La brecha de género en jubilaciones y pensiones, los casos de Argentina, Brasil, Chile y Uruguay. Serie Asuntos de Género. Santiago de Chile: CEPAL y Cooperación Española, 20ı6. https://repositorio.cepal.org/bitstream/handle/ı I 362/40650/2/S I600929_es.pdf

Amín, Mohammad, Asif Islam y Alena Sakhonchik. "Does paternity leave matter for female employment in developing economies? Evidence from Firm Data". Policy Research Working Paper 7588. World Bank Group, 2016. http://documents.worldbank.org/curated/ en/r 2422 I 468 I $96762078 / \mathrm{pdf} / \mathrm{WPS}_{75}$ 88.pdf

Arnarson, Björn Thor y Aparna Mitra. "The Paternity Leave Act in Iceland: implications for gender equality in the labour market". Applied Economics Letters I $7,{ }^{\circ} 7$ (2010): 677-680. https://doi.org/Io. IO80/I 3504850802297830 king Parents Need Paid Timeoff. The Clearinghouse on International Developments in Child, Youth \& Family Policies" (Issue brief, Columbia University, 2002); Mohammad Amin, Asif Islam y Alena Sakhonchik, "Does Paternity Leave Matter for Female Employment in Developing Economies? Evidence from Firm Data" (Policy Research Working Paper 7588, World Bank Group, 20r6) y, en especial, Björn Thor Arnarson y Aparna Mitra, "The Paternity Leave Act in Iceland: Implications for Gender Equality in the Labour Market", App. Econ. Letters I 7, n. ${ }^{\circ}$ (2010): 677-680, para el caso islandés. Islandia se caracteriza de los demás países del mundo por la licencia de paternidad más extensa, de I3 semanas. 
Arango, Luis Eduardo y Ana María Ríos. "Duración del desempleo en Colombia: género, intensidad de búsqueda y anuncios de vacantes”. En Desempleo femenino en Colombia. Editado por Luis Eduardo Arango, Francesca Castellani y Eduardo Lora, I35-i69. Bogotá: Banco de la República у вІD, 20 I6. http://babel.banrepcultural.org/cdm/ref/collection/pi 7054collı 8/ $\mathrm{id} / 285$

Arza, Camila. "The gender dimensions of pension systems: Polices and constraints for the protection of older women". Discussion paper I, Progress of the World's Women 201520I6, UN Women, 20I5. http://socialprotection-humanrights.org/wp-content/uploads /2015/o8/ARZA-Fin.pdf

Bosch, Mariano, Solange Berstein, Francesca Castellani, María Laura Oliveri y Juan Miguel Villa. "Diagnóstico del sistema previsional colombiano y opciones de reforma". Nota Técnica 825, Unidad de Mercados Laborales y Seguridad Social, BID, 20I 5. https://goo.gl/cjicqU

Choi, Jongkyun. “The Role of Derived Rights for Old-age Income Security of Women”. Employment and Migration Working Papers 43, oecd Social, París: oecd Publishing, 2006. http:// dx.doi.org/ıo. I 787/350882777255

Comisión Asesora Presidencial Sobre el Sistema de Pensiones-Comisión Bravo. Comisión presidencial pensiones: informe final. 20I5. http://www.comision-pensiones.cl/Informe_final_ CP_2015.pdf

D'Addio, Anna Cristina. "Pensions entitlements of women with children: The role of credits within pension systems in oEcD and eu countries". En Nonfinancial Defined Contribution Pension Schemes in a Changing World, Volume 2: Gender, Politics and Financial Stability. Editado por Robert Holzmann, Edward Palmer y David Robalino, Washington D.C.: The World Bank, 20I3. https://www.diva-portal.org/smash/get/diva2:576653/FULLTEXTOI.pdf

Farné, Stefano. "Pensiones y mujeres". El Espectador, 28 de febrero de 201 5. https://www.elespectador.com/noticias/economia/pensiones-y-mujeres-articulo-54662 I

Fajnzylber, Eduardo. "Gender Policy and Pensions in Chile". En Nonfinancial Defined Contribution Pension Schemes in a Changing World, Volume 2: Gender, Politics and Financial Stability. Editado por Robert Holzmann, Edward Palmer y David Robalino. Washington D.C.: The World Bank, 20I3. https://www.diva-portal.org/smash/get/diva2:576653/FulLteXTor.pdf

Favreault, Melissa y Eugene Steuerle. "Social Security Spouse and Survivor Benefits for the Modern Family". CRR wP 2007-7. Chestnut Hill: Center for Retirement Research at Boston College, 2007. http://crr.bc.edu/wp-content/uploads/2007/02/wp_2007-7-508.pdf

Federación Internacional de Administradoras de Fondos de Pensions (fiap). "Analysis of the Proposal for Unisex Mortality Tables for Calculating Old-Age Pensions in the Individually Funded System”. Pension Notes I 5, 20 I 7. https://www.fiapinternacional.org/wp-content/ uploads/20r6/or/Note-I 5-Unisex-Mortality-Tables.pdf

Fornero, Elsa. "Chapter I r. Comment". En Nonfinancial Defined Contribution Pension Schemes in a Changing World, Volume 2: Gender, Politics and Financial Stability. Editado por Robert Holzmann, Edward Palmer y David Robalino. Washington D. C.: The World Bank, 2013. https://www.diva-portal.org/smash/get/diva2:576653/FULLTEXTOI.pdf

Hotchkiss, Julie y Melinda Pitts. "The role of labor market intermittency in explaining gender wage differentials". The American Economic Review 97, n. ${ }^{\circ}$ 2, (2007): 4I 7-42 I. http://www. jstor.org/stable/30034487 
Jacobsen, Joyce y Laurence Levin. “Effects of Intermittent Labor Force Attachment on Women's Earnings". Monthly Labor Review I 8, (I995): I4-I9. https://www.bls.gov/mlr/ı995/o9/ art2 full.pdf

James, Estelle. "Gender in the (Nonfinancial) Defined Contribution World: Issues and Options". En Nonfinancial Defined Contribution Pension Schemes in a Changing World, Volume 2: Gender, Politics and Financial Stability. Editado por Robert Holzmann, Edward Palmer y David Robalino. Washington D.C.: The World Bank, 2013. https://www.diva-portal.org/smash/get/ diva2:576653/FULLteXTor.pdf

Kamerman, Sheila y Shirley Gatenio. "Mother's day: More than candy and flowers, working parents need paid timeoff. The clearinghouse on international developments in child, youth \& family policies". Columbia University, spring 2002.

Klerby, Anna, Bo Larsson y Edward Palmer. "To share or not to share: That is the question”. En Nonfinancial Defined Contribution Pension Schemes in a Changing World, Volume 2: Gender, Politics and Financial Stability. Editado por Robert Holzmann, Edward Palmer y David Robalino. Washington D.C.: The World Bank, 2013. https://www.diva-portal.org/smash/get/ diva2:576653/Fulltextor.pdf

Lams, Howard M., Gayle L. Reznik y Christopher R. Tamborini. "Earnings sharing in Social Security: Projected impacts of alternative proposals using the mint Model". The Social Security Bulletin 69, n. ${ }^{\circ}$ I (2009): I-I 7. https://www.ncbi.nlm.nih.gov/pubmed/I9579528

López, Hugo y Francisco Lasso. "Diferencias por sexo en los flujos de trabajadores entre estados laborales y el futuro laboral de las mujeres colombianas". En Desempleo femenino en Colombia. Editado por Luis Eduardo Arango, Francesca Castellani y Eduardo Lora. Bogotá: Banco de la República y вID, 20г6. http://babel.banrepcultural.org/cdm/ref/collection/ pr 7054 collis $8 / \mathrm{id} / 285$

Monroy, Viviana y María Alejandra Olarte. "Estudio sobre el comportamiento de la división del trabajo en el hogar: particularidades de género para Colombia". En INVESTIGAS, siete estudios realizados a partir de la encuesta nacional de uso del tiempo, Colombia 20 I 2-2013. Bogotá: DANE y UNFPA, 2OI 5. https://www.dane.gov.co/files/investigaciones/boletines/ENUT/INVESTIGAS_ Siete_estudios_ENUT.pdf

Organización Internacional del Trabajo-ort. Seguridad social: un nuevo consenso. Ginebra: огт, 2002. http://www.ilo.org/wcmsp5/groups/public/---ed_protect/---soc_sec/documents/publication/wcms_220095.pdf

Organisation for Economic Co-operation and Development (oECD). Pensions at a Glance 2015: OECD and G2o indicators. París: oECD Publishing, 201 5. http://dx.doi.org/IO.I 787 /pension_ glance-2015-en

Ramírez, Natalia, Ana María Tribín y Carmiña O. Vargas. "Maternidad y mercado laboral: el impacto de la legislación”. En Desempleo femenino en Colombia. Editado por Luis Eduardo Arango, Francesca Castellani y Eduardo Lora. Bogotá: Banco de la República y вID, 20 I6. http://babel.banrepcultural.org/cdm/ref/collection/pi 7054coll I 8/id/285

Rossin-Slater, Maya. "Maternity and Family Leave Policy." IzA Discussion Paper ı0500. Bonn: 20I 7. https://ssrn.com/abstract=2903 I 22

Sociedad Silva Santos. "Acciones con perspectiva de género en la seguridad social. Actualización de informe". Asesoría General en Seguridad Social, Comentarios de Seguridad Social n. ${ }^{\circ}$ 5 I, 2016. 
Stählberg, Ann-Charlotte. "Chapter I I: Comment". En Nonfinancial Defined Contribution Pension Schemes in a Changing World, Volume 2: Gender, Politics and Financial Stability. Editado por Robert Holzmann, Edward Palmer y David Robalino. Washington D.C.: The World Bank, 20I3. https://www.diva-portal.org/smash/get/diva2:576653/Fulltextor.pdf 